\section{References}

AMERICAN PSYCHIATRIC ASSOCIATION (1987) Diagnostic and Statistical Manual of Mental Disorders (3rd edn, revised) (DSM-III-R). Washington DC: APA.

CITROME, L., GREEN, L. \& FOST, R. (1994) Length of stay and recidivism on a psychiatric intensive care unit. Hospital and Community Psychiatry, $\mathbf{4 5}$, 74-76.
HAFNER, R. J., LAMMERSMA, J., FERRIS, R., et al (1989) The use of seclusion: a comparison of two psychiatric intensive care units. Australian and New Zealand Journal of Psychiatry, 23, 235-239.

HYDE, C. E. \& HARROWER-WILSON, C. (1996) Psychiatric intensive care in acute psychosis. International Clinical

Psychopharmacology, 11(suppl. 2), $61-65$.
MICHALON, M. \& RICHMAN, A. (1990) Factors affecting length of stay in a psychiatric intensive care unit. General Hospital Psychiatry, 2, 303-308.
RACHLIN, S. (1973) On the need for a closed ward in an open hospital: the psychiatric intensive care unit. Hospital and Community Psychiatry, 24 829-833.

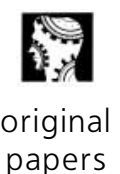

papers

Mairead Dolan MRCPsych, PhD, Edenfield Centre, Mental Health Services of

Salford, Bury New Road, Prestwich, Manchester M25 3BL, Alan Lawson BSC Mathematics, MSc Social Research, Formerly Researcher, Edenfield Centre,

Prestwich Hospital

\title{
A survey of forensic psychiatry teaching in UK medical schools
}

\author{
AIMS AND METHOD \\ To survey forensic psychiatry \\ teaching in UK medical schools. A \\ questionnaire was sent to all 24 \\ deans.
}

\section{RESULTS}

Twenty-one schools responded, 15 (71\%) provided forensic psychiatry teaching. Thirteen organised one or more lectures and 13 organised visits to forensic psychiatric settings, predominantly high security hospitals, but these were usually only available to a proportion of the students in each year. Clinical placements, seminars or workshops and tutorials or supervisions were each arranged in about half of the schools. Only four schools offered special study modules (SSMs).

\section{CLINICAL IMPLICATIONS}

Forensic psychiatry teaching would benefit from further development, with additional use being made of medium secure hospital units and prisons for the purposes of clinical placements and visits. More SSMs should be organised.
In the UK the traditional undergraduate medical curriculum has undergone major revision and change over the past decade. The General Medical Council (GMC), when proposing what form this change should take, recommended that universities should develop a 'core curriculum' that all students would follow, together with a programme of 'special study modules' (SSMs), during which students could study subjects selected by them in more depth (GMC, 1993). Psychiatry is a component of all undergraduate medical curricula (Davies \& McGuire, 2000). As each medical school determines the length of its own mental health attachment, as well as how it is constituted, the actual programmes differ considerably.

Forensic psychiatry has much to offer the medical undergraduate. The sub-speciality is able to provide students with basic education and training in psychiatry. Moreover, it has the potential to be able to teach many transferable skills, such as the principles involved in the treatment of chronic conditions, which are relevant to other areas of the practice of medicine (Reiss \& Meux, 2000). In addition, the discipline needs to encourage recruitment if it is to maintain its expansion and meet the demands placed upon it by both patients and society. Educational initiatives at medical student level may well be effective in stimulating motivation towards a career in the sub-speciality (Thomson et al, 1999). In this light we decided to conduct a national survey of the teaching that is provided to medical students in forensic psychiatry.

\section{Method}

A specially designed questionnaire was sent to the dean of each of the 24 medical schools in the UK. A reminder was sent if there was no response after 3 months. If no reply was received after 3 more weeks then direct telephone calls were made to the appropriate office at each medical school and the questionnaire was faxed or sent again if necessary.

The questionnaire focused on the method of delivery and amount of teaching in forensic psychiatry that was provided. A contact at the medical school was requested in case any details required clarification.

The following terms were defined in the questionnaire:

(a) lecture - large group teaching with limited potential for interaction

(b) seminar or workshop - smaller group learning with opportunity for interaction and /or a practical aspect

(c) tutorial or supervision - very small group, teaching learning techniques or looking at work in progress

(d) clinical placement - student attached to a forensic unit or team for a period of longer than 1 day

(e) visit - student attends a unit or team for up to 1 day.

\section{Results}

Twenty-one responses were received, an $87 \%$ response rate. These medical schools taught a total of 4026 
medical students in each year. Fifteen medical schools (71\% of those who replied) offered some teaching in

original papers 4026 students). The formats of the teaching they forensic psychiatry (representing 2966 students, 74\% of provided are summarised in Table 1.

\section{Lectures}

Thirteen medical schools ( $87 \%$ of the schools that taught forensic psychiatry) provided one or more formal lectures (mode 1; median 2.0; range 1-5), usually lasting 1 hour, on forensic psychiatric topics. In eight of these medical schools a professor, senior lecturer or consultant in forensic psychiatry gave the lectures, in the other six schools the lectures could also be delivered by university lecturers or specialist registrars. The lectures were mostly either on the general topic of forensic psychiatry or about the Mental Health Act.

As shown in Table 1, for one medical school a single lecture was the only teaching in forensic psychiatry that was provided.

\section{Seminars/workshops}

In seven of the schools (47\%) the teaching included one or two seminars or workshops (mode 1; median 1.0), the most common length was 1.5 hours (range 1-8 hours). At three schools the seminars/workshops were given by a senior lecturer or consultant in forensic psychiatry, while in the remaining four the seminars/workshops could also be given by a lecturer or specialist registrar, and at one of them a forensic nurse. All except one of the schools conducted seminars only on the basic topic of forensic psychiatry, as well as the Mental Health Act. The remaining school had a broader range of subjects including areas such as treating patients against their will and ethical issues.

Schools that provided teaching in the form of seminars or workshops always provided teaching in another modality as well.

\section{Tutorials/supervisions}

Five schools provided one or two tutorials or supervisions (mode 1; median 1.0) that lasted between 1 and 1.5 hours (mode 1; median 1.5). The topics discussed included antisocial behaviour and personality disorder. None of the medical schools provided students with teaching in forensic psychiatry that consisted of only a tutorial/ supervision.

\section{Clinical placements}

Six medical schools sent their students on clinical placements that were supervised by consultants or professors in forensic psychiatry. The most common length of placement was 4 weeks (range 4-7 weeks).

Two medical schools sent students to medium secure units only. The other four placed students in a variety of settings including high and medium secure hospitals, locked wards, prisons and courts and community forensic services. A total of 1240 students attended medical schools that provided clinical placements. However, only 606 students attended the placements. The locations of the placements were: high security hospital, 254 (42\%); medium secure hospital unit 64 (11\%); prison 92 (15\%); locked ward 90 (15\%); community forensic services 50 (8\%); open ward $44(7 \%)$; and court $12(2 \%)$

\section{Visits}

Thirteen medical schools (87\%) sent their students on visits to forensic settings. Three organised visits to high security settings only, two to medium secure settings only and one university sent students on visits to locked wards only. The remaining seven schools sent their students on visits to various forensic psychiatry settings: prisons, courts, high and medium secure units, locked and open wards and community forensic services.

Table 1. Teaching formats in forensic psychiatry

\begin{tabular}{|lllllll}
\hline Medical school & Lecture(s) & $\begin{array}{l}\text { Seminar(s) or } \\
\text { workshop(s) }\end{array}$ & $\begin{array}{l}\text { Tutorial(s) or } \\
\text { supervision(s) }\end{array}$ & $\begin{array}{l}\text { Placement } \\
\text { (length in weeks) }\end{array}$ & $\begin{array}{l}\text { Visit(s) } \\
\text { (length in h) }\end{array}$ & Social study modules \\
\hline 1 & No & Yes & No & Yes (4) & No & Yes \\
2 & Yes & Yes & No & Yes (5) & Yes (8) & Yes \\
3 & Yes & Yes & No & No & Yes (3) & No \\
4 & Yes & No & No & No & No & No \\
5 & No & No & No & No & Yes (2) & No \\
6 & Yes & No & No & No & Yes (6) & No \\
7 & Yes & No & No & Yes (7) & Yes (2) & Yes \\
8 & Yes & Yes & No & No & Yes (2) & No \\
9 & Yes & No & No & No & Yes (2) & No \\
10 & Yes & Yes & Yes & Yes (4) & Yes (1) & No \\
11 & Yes & No & No & Yes (5) & Yes (3) & No \\
12 & Yes & Yes & Yes & Yes (4) & Yes (1) & Yes \\
13 & Yes & No & Yes & No & Yes (1.5) & No \\
14 & Yes & No & Yes & No & Yes (4) & No \\
15 & Yes & Yes & Yes & No & & \\
& & & & & &
\end{tabular}


A total of 2826 students attended medical schools that provided visits. However, only 1211 students actually attended clinical visits, although some of these went on more than one. The venues for the visits were: high security hospital, 776 students (64\%); locked ward, 220 (18\%); open ward, 205 (17\%); medium secure hospital unit, 110 (9\%); prison, $26(2 \%)$; court, $16(1 \%)$; and community forensic service, $10(1 \%)$.

For one medical school a visit for 30 of the 166 students in the year to a locked ward was the only form of teaching in forensic psychiatry provided.

\section{Special study modules}

Four medical schools offered SSMs in forensic psychiatry. These took place on 1 day each week and were based in high security hospitals, medium secure units or prisons, and often incorporated visits to various other forensic settings. The duration of the shortest SSM was 4 weeks and the longest 12 weeks. Up to seven students could take part in each running of the SSMs, which took place several times each year.

\section{Written work}

Two medical schools asked a total of 10 students to produce written work in forensic psychiatry.

\section{Discussion}

The study's response rate of $87 \%$ was satisfactory. However, less than three-quarters of the responding medical schools actually provided any forensic psychiatry teaching to their students. Even where teaching was organised, in many schools it was often very limited in extent and in nearly all of them activities such as visits and placements were only available to a relatively small proportion of the students in the year.

Visits were organised much more frequently to high security hospitals, locked and open wards, than to medium secure hospital units and non-hospital settings, which might indicate that medium secure units, community resources, courts and prisons are underdeveloped for teaching purposes. In particular, the continuing expansion of medium security services provides a valuable teaching resource, and students should also be encouraged to witness at first hand the high prevalence of psychiatric disorder in prisons (Singleton, et al, 1998).

Only four of the medical schools provided a SSM in forensic psychiatry. Forensic psychiatry is an ideal subject to be studied in this format, which encourages a longitudinal perspective and allows interested students to explore the subject in depth. Further development should be encouraged.

\section{References}

DAVIES, T. \& MCGUIRE, P. (2000) Teaching medical students in the new millennium. Psychiatric Bulletin, 24, 4-5.

GATWARD, R., et al (1998) Psychiatric Morbidity among Prisoners in England and Wales. London: HMSO.

GENERAL MEDICAL COUNCIL (1993) Tomorrow's Doctors. London: GMC.

REISS, D. \& MEUX, C. (2000) Education and training in forensic psychiatry. Journal of Forensic Psychiatry, 11, THOMSON, L. D. G., GRAY, C. M. \& HUMPHREYS, M. S. (1999) Medical students' perspective of maximum security psychiatric care. Psychiatric Bulletin, 23, 230-232.

\title{
CLAIRE BALL
}

\section{Attention-deficit hyperactivity disorder and the use of methylphenidate}

\author{
A survey of the views of general practitioners
}

\section{AIMS AND METHOD}

General practitioners (GPs) were surveyed on their experience of and attitudes towards attention-deficit hyperactivity disorder (ADHD) treatment using methylphenidate, and asked about prescribing practice.

\section{RESULTS}

Most GPs have experience of children with ADHD and the use of methylphenidate. The majority felt that it was a drug that should be initiated by a specialist who should continue to provide clinical monitoring, but that primary care could provide ongoing prescribing and physical monitoring. There was a lack of training in this area, with most GPs requesting further training both on ADHD and its management.

\section{CLINICAL IMPLICATIONS}

ADHD is a topical issue both in the health service and with the public. This survey suggests that GPs may be willing to play a role in the management of ADHD once the child has seen a specialist, but that child and adolescent mental health services need to consider how training will be provided.
Attention-deficit hyperactivity disorder (ADHD) (DSM-IV; American Psychiatric Association, 1994) occurs in 3-5\% of school-aged children and the number of referrals to child and adolescent psychiatric services is increasing 